\title{
Stage IIIA Uterine Corpus Cancer AJCC v8
}

National Cancer Institute

\section{Source}

National Cancer Institute. Stage IIIA Uterine Corpus Cancer AJCC v8. NCI Thesaurus.

Code C139807.

Stage IIIA includes: T3a, N0, M0. T3a: Uterine corpus carcinoma or carcinosarcoma with tumor involving the serosa and/or adnexa (direct extension or metastasis). N0: No regional lymph node metastasis. M0: No distant metastasis. (from AJCC 8th Ed.) 